\title{
Sistema de tempo real aplicado a simuladores de Ressuscitação Cardiopulmonar
}

\author{
Rodolfo Rocha Vieira Leocádio ${ }^{1,2, *}$, Alan Kardek Rêgo Segundo ${ }^{1}$, Cibelle Ferreira Louzada²
}

\author{
${ }^{1}$ Departamento de Controle e Automação (DECAT), Escola de Minas, Universidade Federal e Ouro Preto (UFOP), Morro do \\ Cruzeiro, 35400-000 Ouro Preto, MG, Brasil (e-mail: alankardek@ufop.edu.br). \\ ${ }^{2}$ Departamento de Clínicas Pediátrica e do Adulto (DECPA), Escola de Medicina, Universidade Federal e Ouro Preto \\ (UFOP), Morro do Cruzeiro, 35400-000 Ouro Preto, MG, Brasil (e-mail: cibelle.louzada@hotmail.com). \\ *(Tel: +55-31-98807-3747; e-mail: Rodolfo.leocadio@ufop.edu.br).
}

\begin{abstract}
The most common practice related to medical urgency and emergency is Cardiopulmonary Resuscitation (CPR). There were no CPR practices in the training of health professionals until the appearance of realistic mannequins intended for this purpose. Then, the automation of this equipment allowed the continuous improvement of the quality of the maneuvers through real-time feedback. This work implements a Soft-Real-Time in order to present the actions performed on the dummy in a sufficient time interval to perform and correct the maneuver simultaneously. The Arduino platform composes the embedded system and is used together with the Resusci Anne ${ }^{\circledR}$ mannequin. The embedded system is based on the Arduino platform, which encodes the actions performed on the Resusci Anne ${ }^{\circledR}$ mannequin through a Real-Time Scheduling and Multitasking (ARTE). Visual Studio is used to develop the supervisory system, based on CPR teaching methodology, which decodes and present the embedded system information. It was possible to allocate the real-time resources taking into account the requirements of the instrumentation, the embedded system, and the real-time scheduling and multitasking to execute the proposed deadline for the application. In this way, it was possible to make the system achieve real-time requirements and provide feedback to the target audience of this work. The automation of simulators, as presented in this work, brings continuous improvement in the training and training of health professionals, making them able to perform effective maneuvers during CPR.
\end{abstract}

Resumo: A prática mais comum relacionada a urgência e emergência médicas é a Ressuscitação Cardiopulmonar (RCP). Práticas de RCP não foram realizadas na formação de profissionais da área de saúde até o surgimento de manequins realísticos destinados a essa finalidade. Em seguida, a automação desses equipamentos permitiu a melhoria contínua da qualidade das manobras por meio de feedback em tempo real. Neste trabalho, implementa-se um sistema de tempo real de forma que as ações praticadas no manequim são apresentadas em um intervalo de tempo com duração suficiente para realizar e corrigir a manobra simultaneamente. O sistema embarcado foi baseado na plataforma Arduino, que codifica as ações realizadas no manequim Ressusci Anne ${ }^{\circledR}$ por meio de um Escalonador de Tempo Real e Multitarefa (ARTE). O Visual Studio é utilizado para desenvolver um supervisório baseado em metodologia de ensino da RCP, que decodifica e apresenta as informações do sistema embarcado. Foi possível alocar os recursos de tempo real levando-se em consideração as exigências da instrumentação, do sistema embarcado e do escalonador de tempo real e multitarefa para executar o deadline proposto para a aplicação. Desta forma, foi possível fazer com que o sistema atenda aos requisitos de tempo real e forneça feedback ao público-alvo deste trabalho. A automação de simuladores, da forma como apresentado neste trabalho, pode proporcionar melhoria contínua na formação e treinamento dos profissionais da área de saúde, tornando-os mais aptos à realização de manobras efetivas durante a RCP.

Keywords: Real-Time Feedback; Real-Time Scheduling and Multitasking; Embedded System; Medical Urgency and Emergency, Supervisory System.

Palavras-chaves: Feedback em Tempo Real; Escalonador de Tempo Real e Multitarefa; Sistema Embarcado; Urgência e Emergência Médicas; Sistema Supervisório.

\section{INTRODUÇÃO}

Com o avanço tecnológico proporcionado por áreas distintas como automação, mecânica, materiais e eletrônica, foi possível desenvolver simuladores que repetem o comportamento de seres humanos em estado grave ou em risco iminente de morte. Um dos primeiros registros que se tem da automação de simuladores de Ressuscitação Cardiopulmonar (RCP) no cenário mundial é datada no ano de 1972, referente ao trabalho de Patrick e Eisenberg (1972). Esses manequins eram utilizados nos países de primeiro mundo em simulações de Suporte Básico de Vida (SBV). 
Até o ano de 2002, no Brasil, práticas relacionadas a urgência e emergência médicas não eram realizadas na formação de profissionais da área de saúde. O recém-formado era absorvido pelo mercado de trabalho somente com a teoria acerca desse assunto (Fraga et al., 2013).

O cenário atual coloca à disposição dos centros educacionais simuladores que oferecem condições de praticar procedimentos variados. A maioria desses procedimentos são impossíveis de serem realizados sem risco ao paciente.

A prática mais comum relacionada a urgência e emergência médicas é a RCP, constituída de manobras realizadas na tentativa de reanimar uma pessoa vítima de parada cardíaca e/ou respiratória. A técnica tem como finalidade fazer com que o coração e pulmão voltem às suas funções normais, mantendo a oxigenação do cérebro.

A automação dos simuladores para RCP proposta por este estudo envolve a instrumentação de um manequim desenvolvido para essa finalidade. Um sistema embarcado compõe o manequim e tem como objetivo interpretar os sinais gerados por sensores nele instalados e realizar a interface com um PC.

Neste trabalho, as ações praticadas no manequim devem ser apresentadas em um intervalo de tempo suficientemente adequado para que a pessoa que realiza a manobra tenha um feedback, de forma que a mesma consiga corrigir a manobra enquanto a realiza. Assim, um sistema de tempo real é responsável pelo gerenciamento do sistema embarcado.

Automatizar esses simuladores pode proporcionar melhoria continua na qualidade do ensino da área de saúde. Isso torna os profissionais mais aptos à realização de manobras efetivas durante a RCP.

\section{METODOLOGIA}

Nesta seção, os recursos utilizados para a implementação da proposta são apresentados, visando empregar e/ou adequar (quando necessário) os equipamentos necessários à aplicação.

\subsection{Descrição do manequim}

Os profissionais de saúde utilizam manequins em aulas, treinamentos e outras capacitações, com o objetivo de aprender e treinar as manobras de RCP. O manequim automatizado tem como objetivo codificar as ações externas aplicadas para serem interpretadas pelo sistema embarcado.

Neste trabalho, foi utilizado o manequim Resusci Anne SkillReporter ${ }^{\circledR}$, com algumas modificações para atender ao objetivo do estudo. A escolha desse manequim se justifica por ele ser o mais completo em termos de automação para aplicações de RCP.

Os procedimentos realizados durante as manobras estão de acordo com as normas da AHA (2015), AHA (2017) e AHA (2018). O manequim contém em seu interior um sensor de estado da via aérea, um sensor de palpação de pulso, um sensor de posição das mãos e um sensor de profundidade de compressão e volume de insuflação.

Esse conjunto de sensores foram desenvolvidos para treinamento das diretrizes de SBV do ano de 2010. Para atender as diretrizes atuais o último sensor foi substituído por outros dois sensores. A profundidade de compressão até 2015 era de 38 a $51 \mathrm{~mm}$, e a partir daí foi atualizada para 50 a 60 $\mathrm{mm}$. O sensor contido no manequim somente realiza medidas até aproximadamente $60 \mathrm{~mm}$, desta forma não há como simular uma compressão profunda demais, o que causaria lacerações ao coração, comprometendo assim a função do feedback. O novo sensor alcança até $80 \mathrm{~mm}$, por isso consegue simular compressões mais profundas.

A novidade na instrumentação do manequim foi a utilização do sensor ultrassônico HC-SR04 como sensor de profundidade de compressão, do sensor de vazão YF-S201 como sensor de volume de insuflação e da plataforma Arduino como sistema embarcado de tempo real. A utilização do YF-S201 ainda trouxe vantagens de realizar a medida direta do ar que entra no pulmão considerando parâmetros espirométricos.

\subsubsection{Sensor de palpação de pulso}

O sensor de palpação de pulso é exibido na Figura 1a e b, contendo os detalhes sobre o pulso direito (PD) e o pulso esquerdo (PE), respectivamente. O sensor é constituído por contatos Normal Aberto (NA), utilizando polímero flexível, posicionados abaixo da artéria carotídea; e possui trilhas compostas por polímero condutor iônico, também flexível, para não perder a forma anatômica do pescoço.

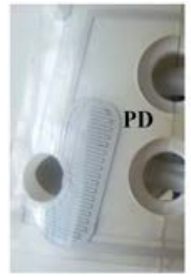

(a)

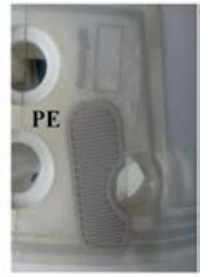

(b)
Fig. 1 Sensor de palpação de pulso.

O objetivo deste sensor é informar se o socorrista está aferindo o pulso carotídeo. Um tempo de $100 \mathrm{~ms}$ para atualizar o estado de PD e PE, considerando o tempo da percepção humana, é adequado para a aplicação. Portanto, foi considerado como o requisito de tempo real para a palpação do pulso carotídeo.

\subsubsection{Sensor de estado da via aérea}

O sensor de estado da via aérea é exibido na Figura 2. Na Figura 2a pode-se visualizar a parte posterior do dispositivo que se movimenta quando a via aérea é aberta, a parte anterior está na Figura 2b. Já na Figura 2c é apresentado a situação de chave aberta no sensor, ou seja, via aérea aberta. O sistema possui um sensor de efeito Hall A1106EUA-T, para detecção dos estados da via aérea. Vale ressaltar que o objeto circular cinza apresentado na Figura 2c é um imã, cujo 
campo magnético não atravessa perpendicularmente o sensor. Na Figura 2d é apresentado a situação de chave fechada no sensor, ou seja, via aérea fechada. Logo, o campo magnético atravessa perpendicularmente o sensor, acionando-o.

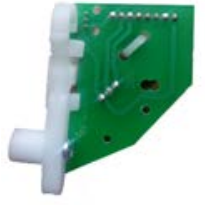

(a)

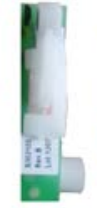

(b)

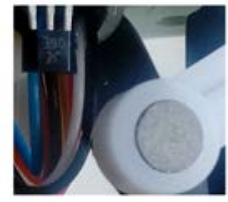

(c)

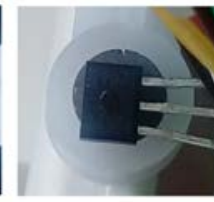

(d)
Fig. 2 Sensor de estado da via aérea.

O objetivo do sensor é gerar informação sobre o estado da via aérea (aberta ou fechada), durante a realização de ventilações de resgate. Esta etapa possui o mesmo requisito de tempo real do anterior.

\subsubsection{Sensor de posição das mãos}

O sensor de posição das mãos é exibido na Figura 3. Vale a pena ressaltar que o objeto apresentado na Figura 3a é a replica anatômica das costelas de um adulto, e serve para orientar o aluno onde deve posicionar suas mãos para realizar RCP. Na Figura 3b, apresenta-se a parte posterior do objeto, e mostra onde o sensor é posicionado e fixado. Já na Figura 3c, mostra-se o elemento sensor, composto por vários contatos dispostos estrategicamente. Por fim, os contatos NA com suas identificações são apresentados na Figura 3d.

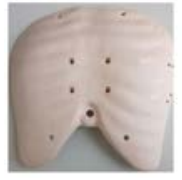

(a)

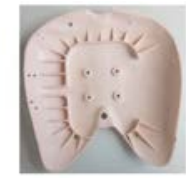

(b)

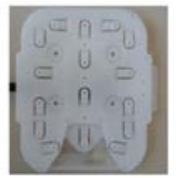

(c)

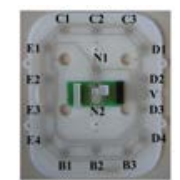

(d)
Fig. 3 Sensor de posição das mãos.

Este sensor tem o objetivo informar se o socorrista tem suas mãos posicionadas corretamente enquanto realiza a manobra de compressão. Os contatos E1-4 identificam mãos posicionadas à esquerda. $\mathrm{O}$ mesmo acontece com os contatos D1-4 (direita), C1-3 (cima), B1-3 (baixo) e N1-2 (mãos posicionadas corretamente). Por último, $\mathrm{V}$ identifica se há peso sobre o tórax, para que não ocorra ventilações e compressões simultâneas. Esta etapa também possui o mesmo requisito de tempo real dos sensores anteriores para cada agrupamento de contatos.

\subsubsection{Sensor de profundidade de compressão}

O módulo ultrassônico HC-SR04, exibido na Figura 4, mede distâncias de 2-400 cm sem contato com a peça móvel, e apresenta incerteza de medição de até $3 \mathrm{~mm}$. O modulo foi posicionado anatomicamente abaixo do órgão cardíaco para medir a profundidade de compressão do tórax.

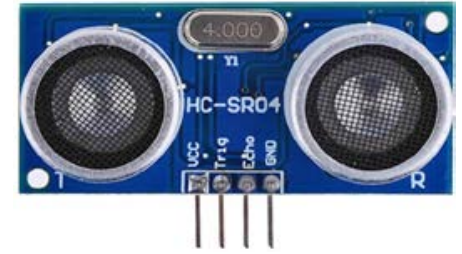

Fig. 4 Sensor de profundidade de compressão.

Cada módulo inclui um transmissor e um receptor ultrassônicos, juntamente com um circuito de controle. Quando um pulso de $10 \mu$ s chega no gatilho, o transmissor do módulo envia 8 ciclos de ultrassom a $40 \mathrm{kHz}$, que é refletido pelo objeto e recebido de volta pelo módulo no pino "Echo". O fabricante sugere usar um ciclo de medição de no mínimo $60 \mathrm{~ms}$ entre as medições, para evitar erros, o que define, assim, o requisito de tempo real do sensor.

\subsubsection{Sensor de volume de insuflação}

O módulo YF-S201, mostrado na Figura 5, é um arranjo simplificado e preciso para quantificação de vazão de água em tubulações. O dispositivo é baseado num sensor de efeito Hall, que gera pulsos proporcionais à vazão de água que passa pelo sensor. $\mathrm{O}$ sensor foi adaptado para informar o volume de ar fornecido aos pulmões durante as ventilações de resgate no trabalho realizado por Leocádio, Segundo e Louzada (2018).

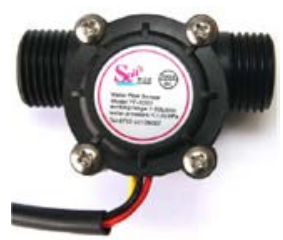

Fig. 5 Sensor de volume de insuflação.

Como o sistema embarcado deve contar pulsos, seu requisito de tempo real passa a ser o mais importante e deve ser tratado assim que ocorrer. Para isso, utilizou-se a interrupção externa do microcontrolador.

\subsection{Sistema Embarcado}

Um sistema embarcado, baseado na plataforma Arduino, foi desenvolvido com o objetivo de interpretar os sinais gerados pelo manequim e fazer a interface entre o manequim e um computador. Essa plataforma, bastante difundida no meio acadêmico, possui código aberto para protótipos eletrônicos, o que garante simplicidade em sua utilização.

Neste projeto, foi selecionada a placa Arduino UNO, por cumprir os requisitos do sistema, ou seja, por possuir: (i) entradas compatíveis com os sensores disponíveis no manequim, de $5 \mathrm{~V}$ e até $40 \mathrm{~mA}$; (ii) comunicação USB, baseada no conversor FTDI; (iii) ambiente de desenvolvimento de código livre, Arduino IDE; e (iv) alimentação por meio de fonte externa com tensão de 7 a 20 V. 


\subsection{Tempo real}

Um sistema de tempo real pode ser definido como qualquer sistema de processamento que deve responder a estímulos de entrada dentro de um período finito e especificado. As principais características dos sistemas de tempo real são tempo de resposta, extrema confiabilidade e segurança, concorrência e interação (Li e Yao, 2003).

Para atingir o propósito dos sistemas de tempo real, podem ser utilizados escalonadores de tempo real e multitarefa como o Real-Time Scheduling and Multitasking (ARTE). O ARTE é uma biblioteca com um conjunto de Interfaces de Programação de Aplicativos (APIs) para usuários desenvolverem suas rotinas. O ARTE é projetado para gerenciar o tempo e agendar a obtenção de respostas em tempo real, fazendo assim a sincronização e gestão de recursos do sistema embarcado (Gardi, 2018). O ARTE deve possuir algumas características em comum com um Sistema Operacional de Tempo Real (RTOS) como programação baseada em prioridades, previsibilidade na sincronização de tarefas e comportamento determinístico (Tan e Tran Nguyen, 2009).

Neste trabalho, foi utilizado um sistema de tempo real embarcado classificado como Soft Real-Time. Em outras palavras, são sistemas de propósitos específicos nos quais os prazos de entrega são importantes, mas continuarão funcionando adequadamente se os prazos não forem atendidos ocasionalmente (Li e Yao, 2003).

As ações praticadas no manequim devem ser apresentadas em um intervalo de tempo suficientemente adequado para que a pessoa que realiza a manobra tenha um feedback, de forma que a mesma consiga corrigir a manobra enquanto a realiza. Portanto, as informações geradas pelo Arduino devem atender a um deadline específico para a aplicação.

O ARTE utilizado foi a biblioteca "Arduino Thread, 2.1.1" desenvolvida por Ivan Seidel, Copyright 2015 do MIT (https://github.com/ivanseidel/ArduinoThread). O hardware não suporta tarefas paralelas, mas a biblioteca consegue otimizar o código gerenciando a execução periódica de várias tarefas e agendar facilmente tarefas entre execuções em um tempo fixo ou variável.

O usuário define um objeto thread para cada tarefa e a biblioteca gerencia sua execução planejada. O ARTE tem a vantagem de permitir o uso de timers e interrupções enquanto executa tarefas e otimiza o uso da memória, já que nenhuma pilha precisa ser alocada por tarefa.

\subsection{Sistema Supervisório (Software)}

O objetivo do sistema supervisório é decodificar as ações externas a uma linguagem universal aos profissionais da saúde, gerando feedback aos usuários. O supervisório foi desenvolvido utilizando o Visual Studio.

O Visual Studio é um ambiente de programação orientado a objetos que pode ser utilizado para criar aplicativos com o intuito de serem utilizados em Windows, iOS e Android. Seu código pode ser desenvolvido em Visual Basic, C, C++, C\# e J\#. Também é um produto de desenvolvimento na área web, usando a plataforma do ASP.NET, como sites e aplicativos móveis. Este trabalho foi desenvolvido utilizando a tecnologia Windows Forms do Visual Studio, implementado com a linguagem de programação C\# no Windows 10.

\section{RESULTADOS E DISCUSSÃO}

Como foi apresentado na Seção 2, o sensor de palpação de pulso é composto de dois contatos, o de via aérea é composto por uma chave e o de posição das mãos é composto por cinco contatos, totalizando oito sensores digitais. O sensor de profundidade de compressão e o de volume de insuflação tem resposta em largura de pulso.

Utilizando recursos de tempo real, foi possível executar o deadline apresentado na Figura 6. O deadline fornece informações sobre o tempo que cada tarefa leva para ser executada e o tempo de execução de cada tarefa. É importante ressaltar que cada sensor envia dados em intervalos de tempo distintos, de acordo com suas exigências de monitorização. Logo, o supervisório apresenta a resposta em um tempo compatível com a dinâmica da manobra em relação à percepção da pessoa que a realiza, considerando que ela precisa se orientar na execução das mesmas.

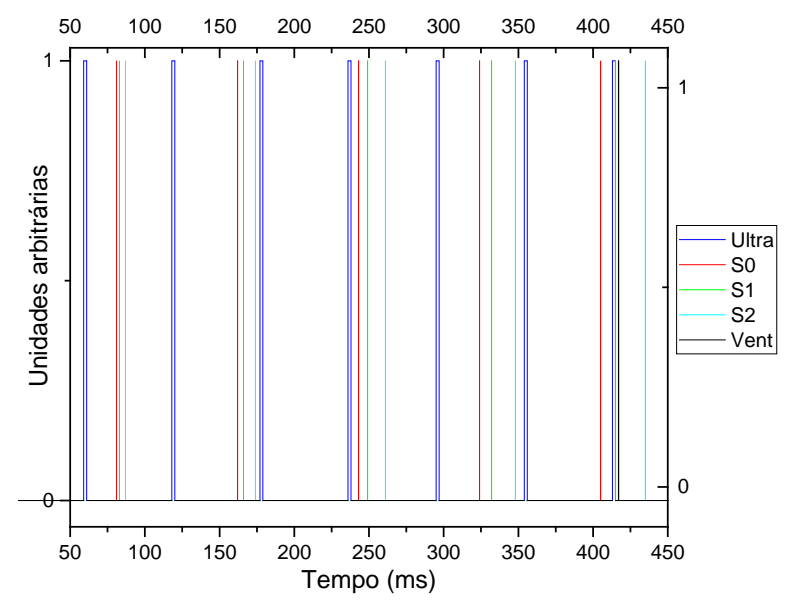

Fig. 6 Deadline do sistema.

A tela principal do supervisório é apresentada na Figura 7a e as respostas dos sensores são representadas de forma gráfica. Na Figura 7b, exemplifica-se uma pessoa realizando a manobra de RCP enquanto observa seu desempenho com a tela exibida na Figura 7a.

O Arduino consegue processar esses sinais de forma eficiente e rápida, todavia, o ponto crítico desta aplicação é justamente o envio dos dados gerados pela thread Ultra para o Visual Studio. Devido à maior periodicidade dessa thread, o buffer de comunicação pode ser sobrecarregado, caso a implementação não considere os requisitos de tempo real.

A comunicação foi feita utilizando-se a porta USART (Universal Synchronous Asynchronous Receiver Transmitter), em conjunto com um conversor FTDI presente na placa, por meio da USB. A velocidade de comunicação foi estabelecida em 2 Mbps. Além do dado, ainda é preciso 
enviar um caractere separador juntamente com um caractere identificador. Os sensores digitais foram codificados em agrupamentos contendo três sensores cada um, e sua informação precisa de 3 bytes e $13 \mu$ s para ser enviada, com periodicidade de 81, 83 e 87 ms para cada agrupamento. Já a informação da profundidade de compressão precisa de 4 bytes e $17 \mu$ s para ser enviada, com periodicidade de $61 \mathrm{~ms}$. Por último, a informação sobre o volume de insuflação necessita de 5 bytes e $21 \mu$ s para ser enviada, sua periodicidade é indeterminada. As tarefas enviam essas informações de acordo com a organização do ARTE, que otimiza a comunicação a ponto de não precisar enviar todas essas informações em um único pacote de dados, o que seria uma desvantagem nesta aplicação.
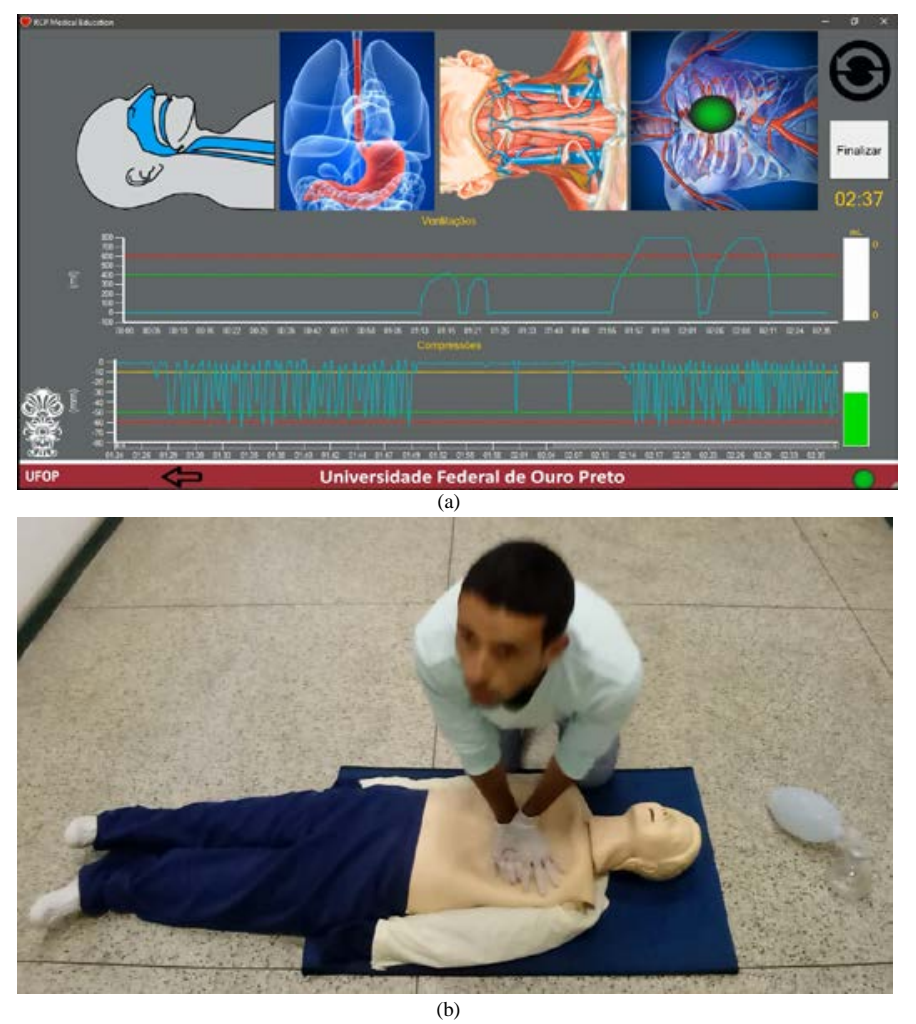

Fig. 7 Tela do Sistema Supervisório (a) produzida pela manobra de RCP realizada (b).

Os agrupamentos de sensores digitais enviam informação a cada 80 ms, pois este se mostrou eficiente para a aplicação. As tarefas dos sensores digitais gastam $50 \mu \mathrm{s}$ para serem executadas, no pior caso. Tentando evitar tempos iguais de execução, como ocorreu em 415 ms na Figura 1, foram escolhidos os tempos de 81, 83 e $87 \mathrm{~ms}$ para os agrupamentos, representados pelas tarefas S0, S1 e S2, respectivamente. Uma variável contendo a situação do estômago durante a ventilação é transmitida em um desses agrupamentos.

A tarefa do sensor ultrassônico ("Ultra”) é a que leva mais tempo de execução: 2 ms. Seguindo a orientação do fabricante, a leitura desse sensor é feita a cada $61 \mathrm{~ms}$. Vale a pena ressaltar que o fabricante propõe $60 \mathrm{~ms}$, mas foi escolhido 61 ms, tentando evitar tempos iguais de execução, como no anterior.
A leitura do sensor de ventilação é feita utilizando uma interrupção ("int”). A Figura 8 mostra que "int” não pode ser executada enquanto "Ultra” estiver em execução. Isso evita instabilidades na leitura e comunicação do sensor ultrassônico. Caso “int” ocorra durante a execução de outra tarefa, ocorre a troca de contexto, pois "int" tem prioridade sob as demais. O tempo para a leitura do sensor de ventilação e cálculo do volume de insuflação para o pior caso é de 400 $\mu \mathrm{s}$.

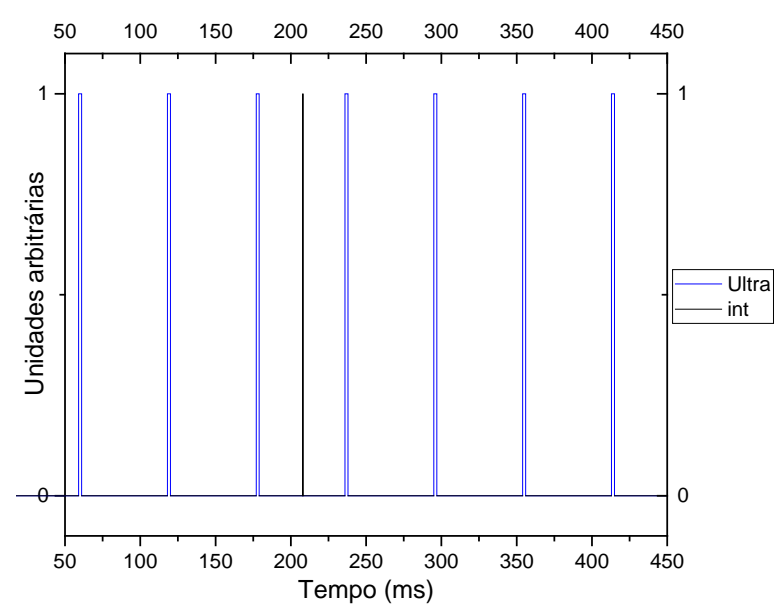

Fig. 8 Exemplo de ocorrência de uma interrupção.

Devido a limitações construtivas do sensor de ventilação, após uma medida não ocorre o retorno espontâneo para a posição de equilíbrio da medida, ou seja, $0 \mathrm{ml}$. Assim a cada 417 ms uma tarefa é executada para zerar a medida do sensor caso este não esteja fazendo leituras. Esse tempo foi escolhido por meio da realização de testes práticos com o equipamento, sendo que com 417 ms obteve-se melhores resultados. O tempo de execução desta tarefa é de $150 \mu$ s.

Foi possível realizar a comunicação do supervisório com os sensores digitais. As informações que o supervisório gera aos profissionais de saúde sobre a situação dos sensores digitais ocorre de forma visual utilizando o recurso de troca de imagens ilustrativas ou sobreposição destas imagens.

A comunicação do supervisório com o sensor ultrassônico também foi implementada. As informações de profundidade de compressão são apresentadas utilizando a ferramenta gráfica em conjunto com a barra de progresso, ambas ferramentas do Visual Studio. O mesmo ocorreu com o sensor da ventilação.

A interrupção tem prioridade de execução, desde que a tarefa Ultra não esteja sendo executada, caso contrário, a prioridade passa a ser de Ultra. Desta forma, o sistema pode apresentar erros somente devido a falhas de comunicação para esses sensores. Como as tarefas dos sensores digitais podem ser interrompidas, estas podem apresentar erros devido a falhas de comunicação e devido a trocas de contexto.

Rotinas para eliminar esses erros foram implementadas no Visual Studio para cada tarefa responsável pelo tratamento dos dados após a comunicação com o sistema embarcado. As leituras com erro dos sensores digitais são desprezadas. Todas as leituras do sensor de profundidade de compressão 
passam por rotinas para identificar se são números de 2 dígitos, caso contrário são desprezadas. O mesmo acontece para o sensor volume de insuflação, mas é verificado se são números de 3 dígitos. Desta forma o supervisório conseguiu atender ao seu objetivo de gerar feedback em um tempo suficiente para o usuário corrigir suas manobras enquanto as realiza.

\section{CONCLUSÕES}

Neste trabalho foi apresentado um simulador para treinamento de RCP juntamente com sua instrumentação necessária a monitorização da manobra. Requisitos de tempo real foram estabelecidos em acordo com as exigências da aplicação e implementadas utilizando um ARTE para sistemas embarcados de tempo real. O deadline de execução foi discutido com base nos requisitos de comunicação serial e de tempo real avaliando se o tempo de resposta do sistema supervisório foi adequado à aplicação.

Foi possível prever o tempo de resposta dos sensores no pior caso, o que proporcionou a interação entre o ARTE e o hardware de acordo com os requisitos do sistema, sendo possível programar de uma maneira abstrata e confiável. Logo, a implementação do Sistema de Tempo Real foi realizada com extrema confiabilidade e segurança.

Tão logo o supervisório recebe os dados, ele os decodifica e apresenta as imformações em um intervalo de tempo suficientemente pequeno para que os usuários possam corrigir as manobras ao mesmo tempo em que as realizam.

Em trabalhos futuros, pretende-se alterar a forma de comunicação de USB para wi-fi, pois vai trazer mais realismo às simulações. O desafio será fazer a comunicação wi-fi cumprir os requisitos de tempo real. Pretende-se, ainda, concluir o feedback de estatísticas da simulação e de avaliação para finalizar com a validação do software como um sistema metodológico para educação.

\section{AGRADECIMENTOS}

Os autores agradecem ao Laboratório de Controle e Automação Multiusuário (LabCAM) da Escola de Minas/UFOP e ao Laboratório de Práticas Simuladas (LAPS) do EMED/UFOP, pela infraestrutura oferecida; ao Laboratório de Saúde Coletiva (LABSC) do EMED/UFOP, por emprestar o espirômetro; e à Universidade Federal de Ouro Preto (UFOP) e à Coordenação de Aperfeiçoamento de Pessoal de Nível Superior (CAPES), pelo apoio financeiro.

\section{REFERENNCIAS}

Association, A. H. (2015) 'Atualização das Diretrizes de RCP e ACE: Destaques da American Heart Association 2015', American Heart Association, p. 36. doi: 10.1161/CIR.0000000000000253.

Association, A. H. (2017) 'Resumo dos principais pontos de discussão e alterações nas atualizações das diretrizes de 2017', atualizações específicas do SBV, p. 2. Available at: https://eccguidelines.heart.org/wp-content/uploads/2017/12/

\section{7-Focused-Updates_Highlights_PTBR.pdf.}

Association, A. H. (2018) 'Destaques das atualizações focadas em Recomendações de 2018 da American Heart Association para RCP e ACE', pp. 1-8.

Fraga, G. P. et al. (2013) 'A situação do ensino de urgência e emergência nos cursos de graduação de medicina no Brasil e as recomendações para a matriz curricular', 10 Anos Das Diretrizes Curriculares Nacionais Do Curso De Graduação Em Medicina, Único, pp. 41-56. Available at: http://abemeducmed.org.br/wp-content/uploads/2016/06/Projetos_ABE M_Diretrizes_Curriculares_Cap3.pdf.

Gardi, M. (2018) Design and Development of RTOS (Scheduler) framework with CPU components analysis (PulseAT implementation) of DAEbot's Health Controller.

Leocádio, R. R. V., Segundo, A. K. R. and Louzada, C. F. (2018) 'Sensor for Measuring the Volume of Air Supplied to the Lungs of Adult Mannequins in Ventilation Maneuvers during Cardiopulmonary Resuscitation', Proceedings, 4(1), p. 39. doi: 10.3390/ecsa-5-05724.

Li, Q. and Yao, C. (2003) Real-Time Concepts for Embedded Systems. CMP Books. doi: 10.1016/j.emc.2005.03.008.

Patrick, H. and Eisenberg, L. (1972) 'An Electronic Resuscitation Evaluation System', IEEE Transactions on Biomedical Engineering, BME-19(4), pp. 317-320. doi: 10.1109/TBME.1972.324078.

Tan, S. L. and Tran Nguyen, B. A. (2009) 'Survey and performance evaluation of real-time operating systems (RTOS) for small microcontrollers', IEEE Micro, pp. 1-14. doi: $10.1109 / \mathrm{mm} .2009 .56$. 